Check for updates

Cite this: RSC Adv., 2018, 8, 37295

Received 13th August 2018 Accepted 15th October 2018

DOI: $10.1039 / c 8 r a 06801 a$

rsc.li/rsc-advances

\section{1,2-Benzoxathiin-4(3H)-one 2,2-dioxide - new enol nucleophile in three-component interaction with benzaldehydes and active methylene nitriles $\uparrow$}

\author{
Galyna V. Grygoriv, (D) a Dmitry A. Lega, (D) a Valentin P. Chernykh, (D) \\ Lucjusz Zaprutko, (D) *b Andrzej K. Gzella, (D) ${ }^{\mathrm{b}}$ Anna Pawetczyk (D) ${ }^{\mathrm{b}}$ \\ and Leonid A. Shemchuk (D)*a
}

\begin{abstract}
The reactivity of 1,2-benzoxathiin-4(3H)-one 2,2-dioxide was studied in multicomponent type reactions for the first time, namely, in a three-component interaction with active methylene nitriles and aromatic aldehydes in order to construct condensed 2-amino-4H-pyran derivatives. The reaction outcome strongly depended on the nature of an active methylene nitrile and an arenecarbaldehyde. Application of malononitrile resulted in novel 2-amino-4-aryl-4H-pyrano[3,2-c][1,2] benzoxathiine-3-carbonitrile 5,5dioxides in most cases, whereas the utilization of ethyl cyanoacetate resulted in a complex mixture of products. In the last case, three different products were isolated depending on the arenecarbaldehyde used, namely ethyl 2-amino-4-aryl-4H-pyrano[3,2-c][1,2] benzoxathiine-3-carboxylate 5,5-dioxides, ethyl 2-cyano-3-arylacrylates, and salts of 3,3'-(arylmethylene)bis(4-hydroxybenzo[e][1,2] oxathiine 2,2dioxides). Attempts to obtain separately ethyl 2-amino-4-aryl-4H-pyrano[3,2-c][1,2]benzoxathiine-3carboxylate 5,5-dioxides enabled us to propose reaction pathways leading to these products. The salts were obtained for the first time. The preparative method for the synthesis of triethylammonium salts of 3,3'-(arylmethylene)bis(4-hydroxybenzo[e][1,2]oxathiine 2,2-dioxides) was proposed by the direct interaction of 1,2-benzoxathiin-4(3H)-one 2,2-dioxide with arenecarbaldehydes. The application of ammonium acetate as a catalyst allowed us to synthesize 7-aryl-7,14-dihydrobenzo[5,6][1,2]oxathiino [4,3-b]benzo[5,6][1,2] oxathiino[3,4-e]pyridine 6,6,8,8-tetraoxides containing a novel heterocyclic system. These facts, combined with our past investigations, allowed us to assert that the reactivity of enol nucleophiles that include the $\mathrm{COCH}_{2} \mathrm{SO}_{2} \mathrm{X}$ fragment has not been reported previously.
\end{abstract}

\section{Introduction}

To date, synthetic organic chemistry does a great deal of work and it is difficult to find a relatively simple organic compound which is poorly studied in respect of its chemical and/or biological properties. Nevertheless, 1,2-benzoxathiin-4(3H)-one 2,2dioxide (1) turned out to be one of these 'dark places' in medicinal chemistry (Fig. 1).

Despite the presence of the synthetically attractive $\mathrm{COCH}_{2} \mathrm{SO}_{2}$ moiety in its structure, the chemistry of the 1,2-benzoxathiin$4(3 H)$-one 2,2-dioxide core is scarcely investigated. There are only a few reports dedicated to the study of its chemical

\footnotetext{
${ }^{a}$ National University of Pharmacy, Pushkinska Str. 53, Kharkiv, 61001, Ukraine. E-mail: orgchem@nuph.edu.ua

${ }^{b}$ Poznan University of Medical Sciences, Pharmaceutical Faculty, Organic Chemistry Department, 6 Grunwaldzka Str, 60-780 Poznan, Poland

$\dagger$ Electronic supplementary information (ESI) available: Full spectroscopic data $\left({ }^{1} \mathrm{H}\right.$ and ${ }^{13} \mathrm{C}$ NMR, IR, MS) for compounds 4, 6, 7 and 9 as well as X-ray crystallographic data for compounds $4 \mathbf{b}$ and 6a. CCDC 1585657 and 1585656. For ESI and crystallographic data in CIF or other electronic format see DOI: 10.1039/c8ra06801a
}

transformations and are mainly disclosed in the works of Löwe et al. ${ }^{1}$ and also some other researchers. ${ }^{2}$ However, all of them are based on multi-stage processes, which reduce their attractiveness in terms of creating combinatorial libraries of new compounds. ${ }^{3}$ This could be related to the fact that the multicomponent processes are often associated with ambiguous reaction mechanisms, selectivity issues or unexpected outcomes. ${ }^{4}$ However, such reactions still remain the main provider of novel core-structures. ${ }^{5}$

At the beginning of the $20^{\text {th }}$ century the concept of isosterism became relevant in medicinal chemistry and remains

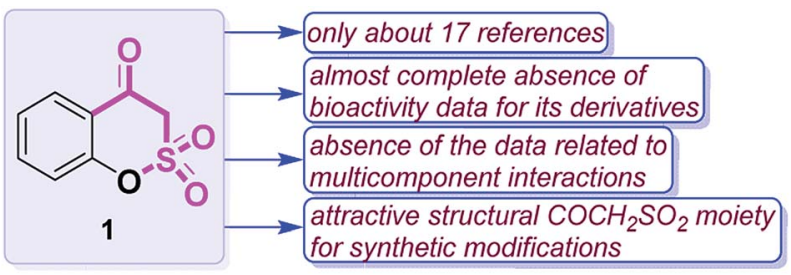

Fig. 1 The main peculiarities of the 1,2-benzoxathiin-4(3H)-one 2,2dioxide (1) core. 

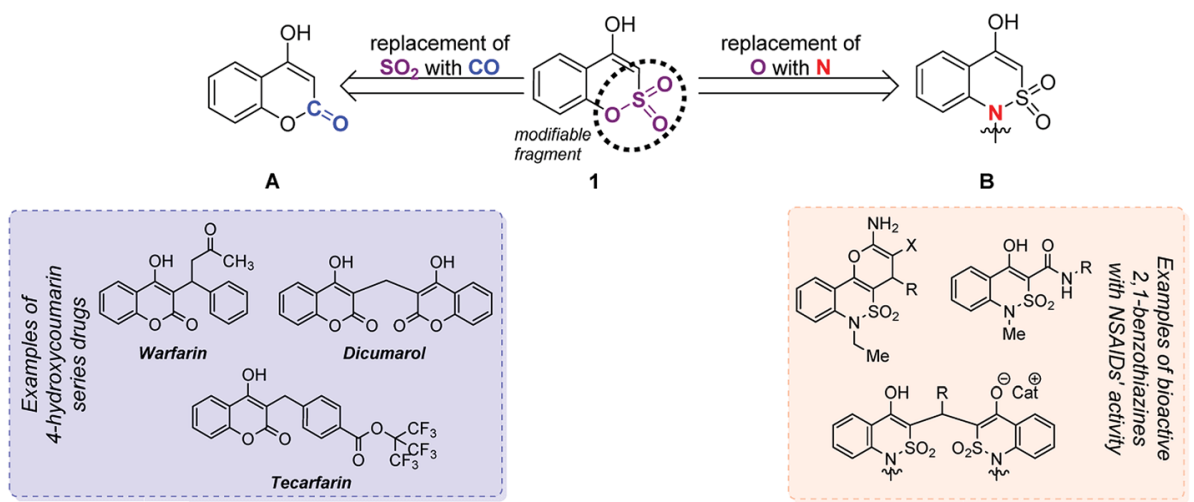

Fig. 2 Isosteric relations of the 1,2-benzoxathiin-4(3H)-one 2,2-dioxide (1) core with 4-hydroxycoumarin $(\mathrm{A})$ and $1 \mathrm{H}$-2,1-benzothiazin-4(3H)one 2,2-dioxide (B) derivatives.

a powerful tool for the purposeful searching of new bioactive substances. ${ }^{6}$ In this regard, the 1,2-benzoxathiin-4(3H)-one 2,2dioxide core can be considered as an isostere for two groups of heterocycles. The first of them comprises such a famous pharmacophore as the 4-hydroxycoumarin core A (Fig. 2). Its derivatives have become well-known drugs and among others are used as anticoagulants by means of vitamin $\mathrm{K}$ inhibiting. ${ }^{7}$ The second group includes 1H-2,1-benzothiazin-4(3H)-one 2,2dioxide $\mathbf{B}$ derivatives, which have recently shown a high effectiveness in the treatment of pain and inflammation and are much stronger NSAIDs compared to the famous drugs Piroxicam ${ }^{\circledR}$ and Meloxicam ${ }^{\circledR}{ }^{8}$ In this way 1,2 -benzoxathiin4(3H)-one 2,2-dioxide (1) conceals inside itself a great potential for drug creation.

Previously, we explored 1H-2,1-benzothiazin-4(3H)-one 2,2dioxide (B) as a synthetic analog of 1,3-dicarbonyls in the multicomponent synthesis of fused 2-amino- $4 \mathrm{H}$-pyrans $\mathbf{E}$ based on the three-component interaction of the former with wide range of aldehydes $\mathbf{C}$ and active methylene nitriles $\mathbf{D}$ (Scheme 1). ${ }^{9,10}$ This allowed us to isolate two product types - 2-amino- $4 H^{-}$ pyrans $\mathbf{E}$ and/or salts $\mathbf{F}$, depending on the starting reagents used and conditions applied in the reaction.

As for 2-amino- $4 \mathrm{H}$-pyrans, they have previously appeared to be a promising chemical class to search among for substances displaying valuable kinds of bioactivity such as anticancer, ${ }^{11}$ antibacterial, ${ }^{12,13}$ anti-rheumatic ${ }^{14}$ and others.

Thereby, as a logical continuation of our research, in this paper we, for the first time, have uncovered the chemical behavior of 1,2-benzoxathiin-4(3H)-one 2,2-dioxide (1) in multicomponent type reactions, particularly in the threecomponent interaction with active methylene nitriles and aromatic aldehydes in order to construct condensed 2-amino$4 H$-pyrans.

\section{Results and discussion}

1,2-Benzoxathiin-4(3H)-one 2,2-dioxide (1) was synthesized by a modified two-stage procedure described in the work ${ }^{15} \mathrm{using}$ methyl salicylate as the starting compound (see Experimental section).

Literature data reveal the application of various reaction conditions and catalysts in the three-component interaction of enol nucleophiles with active methylene nitriles and carbonyl compounds easily leading in most cases to 2 -amino- $4 H$-pyrans irrespective of the reactant nature. ${ }^{16}$ Previously found features gave us an opportunity to predict a non-conventional reactivity of $\mathrm{SO}_{2}$-containing enol nucleophiles in such types of interactions. ${ }^{9,10 b}$ Because of this, first of all we studied the model reaction of 1,2-benzoxathiin-4(3H)-one 2,2-dioxide (1) with malononitrile (3) as an active methylene nitrile and benzaldehyde (2a) as a representative of aromatic aldehydes to find acceptable reaction conditions resulting in the target condensed 2-amino-4-phenyl- $4 H$-pyran-3-carbonitrile 4a (Table 1). Initially, the task was set to find the most suitable catalyst for the reaction using common reaction conditions according to the literature data, ${ }^{16,17}$ specifically the interaction of equimolar quantities of the reagents in ethanol. Results for this step are given in Table 1. As can be seen from the table, without any catalyst the reaction resulted in the formation of fused compound $4 \mathrm{a}$ with only $4 \%$ yield. Thereby, the range of basic and acidic catalysts was tested in order to increase the reaction yield. According to the obtained data, triethylamine turned out

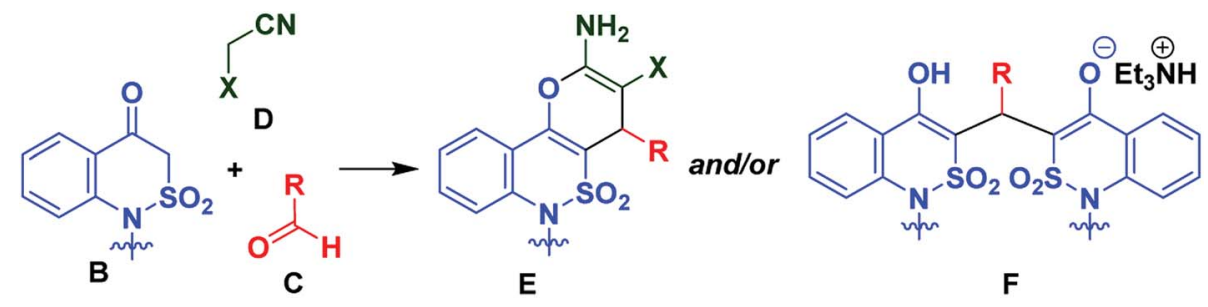

Scheme 1 Previously published results regarding 1H-2,1-benzothiazin-4(3H)-one 2,2-dioxide (B). 
Table 1 Optimization of the reaction conditions and synthesis and yields of annulated 2-amino-4H-pyran-3-carbonitriles 4

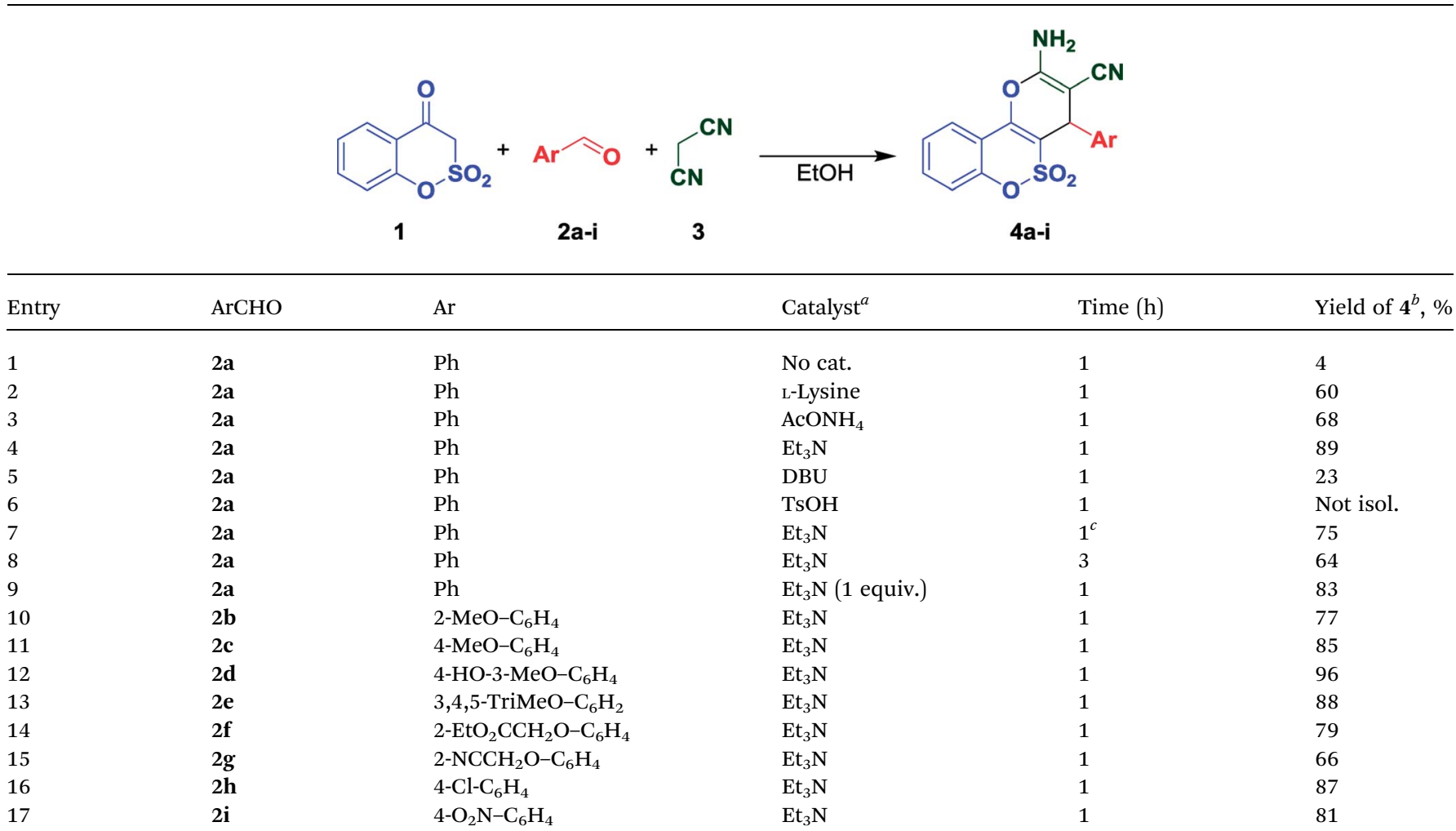

${ }^{a}$ A catalyst was used in the amount of 0.1 equiv. unless otherwise specified. ${ }^{b}$ Isolated yields. ${ }^{c}$ Reaction was carried out at room temperature.

to be the most acceptable catalyst for the tested reaction (Table 1 , entry 4). It should also be noted that the usage of the much stronger base 1,8-diazabicyclo(5.4.0)undec-7-ene (DBU) led to a significant decrease of the 2-amino- $4 H$-pyran 4 a yield. Applying more than 0.1 equiv. of triethylamine as well as prolongation of the reaction time and carrying out of the reaction under room temperature did not result in improving the reaction efficiency. Despite the previously reported successful application of acidic catalysts in the synthesis of 2 -amino- $4 \mathrm{H}^{-}$ pyrans, ${ }^{18,19}$ we failed in our efforts to obtain the target 2-amino$4 H$-pyran 4 a when $p$-toluenesulfonic acid was applied.

According to the data given above, the reflux of equimolar quantities of the reactants in ethanol for $1 \mathrm{~h}$ in the presence of 0.1 equiv. of triethylamine was chosen as the general condition for further investigations. In this regard, a range of substituted aromatic aldehydes was employed at the next step and a series of 2-amino-4-aryl- $4 H$-pyrano[3,2-c] [1,2] benzoxathiine-3-carbonitrile 5,5-dioxides $\mathbf{4 b - i}$ was obtained in high yields (Table 1). All the condensed derivatives 4 were isolated as white or slightly colored crystalline precipitates that can be recrystallized from a mixture EtOH-DMF (5:1, $\mathrm{v} / \mathrm{v})$.

As it turned out, the application of 1,2-benzoxathiin-4(3H)one 2,2-dioxide (1) as an enol nucleophile in the studied reaction had some limitations. Thus, the usage of benzaldehydes containing a strong electron donating substituent (4-dimethylaminobenzaldehyde), or a bulky aromatic residue (9-antraldehyde) resulted in the isolation of only the corresponding
Knoevenagel products ( $\beta$-aryl- $\alpha$-cyanoacrylonitriles) with almost quantitative yields despite our efforts to vary the reaction conditions (reflux for $20 \mathrm{~h}$, application of a range of basic catalysts). This result can be explained not only by means of a significant decrease of the double bond reactivity of the Knoevenagel products in the course of the Michael addition reaction to benzoxathiinone $\mathbf{1}$, but also by a reduced nucleophilicity of 3-C in O-containing benzoxathiinone 1 compared with its N-containing analog - benzothiazinone B. ${ }^{9}$

The next step of the current research was the utilization of ethyl cyanoacetate (5) instead of malononitrile (3) in order to investigate the scope of the studied reaction (Scheme 2). Applying unsubstituted benzaldehyde (2a) as a representative in the three-component interaction made it possible to get a new interesting result in the chemistry of 1,2-benzoxathiin-4 $(3 \mathrm{H})$ one 2,2-dioxide (1). Thus, the use of equimolar amounts of the reagents under reflux for $4 \mathrm{~h}\left(\mathrm{Et}_{3} \mathrm{~N}, 1\right.$ equiv.) led to the isolation of triethylammonium salt $\mathbf{6 a}$ in $17 \%$ yield, which can be easily detected in its ${ }^{1} \mathrm{H}$ NMR spectrum by the presence of an $\mathrm{OH}$ signal as the most downfield peak at about $17 \mathrm{ppm}$.

Similar salts were isolated for the first time under investigations of the three-component condensation involving $N$-ethyl1H-2,1-benzothiazin-4(3H)-one 2,2-dioxide, ethyl cyanoacetate and benzaldehydes. ${ }^{9}$ This allowed us to assert that such chemical behavior is the general property of cyclic $\mathrm{SO}_{2}$-containing enol nucleophiles of the type $\mathrm{XSO}_{2} \mathrm{CH}_{2} \mathrm{CO}$. Because of the low yield of $\mathbf{6 a}$ and still being surprised by its isolation, we additionally analyzed the residue obtained by removing the 


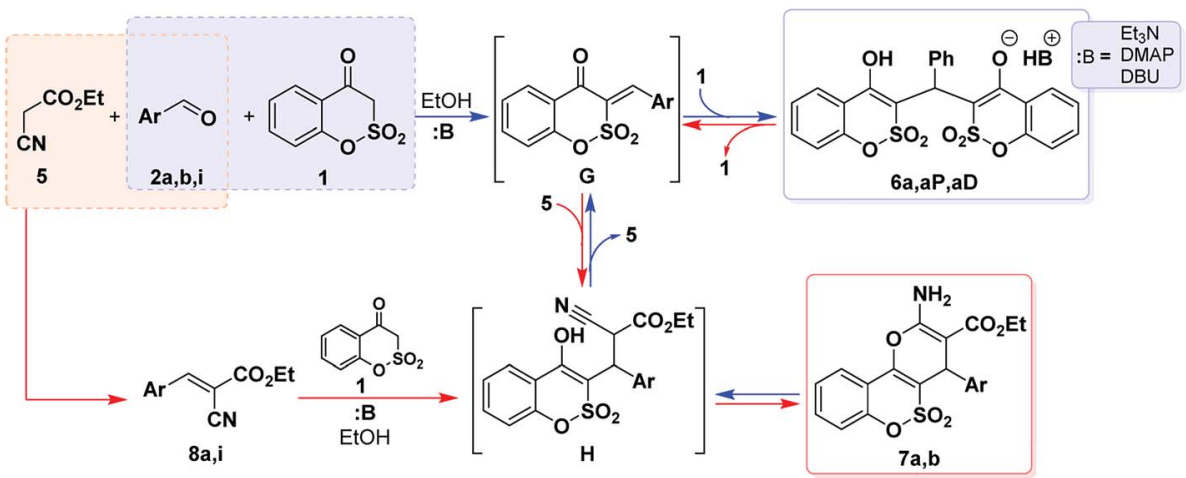

Scheme 2 Three-component reaction involving ethyl cyanoacetate (5).

solvent under reduced pressure using a ${ }^{1} \mathrm{H}$ NMR method. The obtained data evidenced the formation of the desired ethyl 2amino-4-phenyl-4H-pyran-2-carboxylate $7 \mathbf{a}$ along with the salt 6a and ethyl $\alpha$-cyano- $\beta$-phenylacrylate $\mathbf{8 a}$ in an approximately molar ratio $1: 0.6: 0.45$. The application of other benzaldehydes gave us the following results: 2-methoxybenzaldehyde (2b), reacted under the above-mentioned conditions, gave the target 2-amino- $4 H$-pyran $7 \mathbf{b}$ in $27 \%$ yield, while the application of 4-nitrobenzaldehyde (2i) resulted in the isolation of arylidene $8 i$.

It could be assumed that the product mixture, containing $\mathbf{6}$, 7 and 8, was formed in the course of the three-component reaction involving ethyl cyanoacetate 5. In this regard, the isolation of various compositions of the products, depending on the benzaldehyde applied, is related to a different solubility of an individual product in ethanol.

To achieve the goal of the current research we tested several routes, namely the utilization of ethyl cyanoacetate 5 excess, applying different heating modes, variation of catalysts and also a two-component approach ${ }^{20}(\mathbf{8}+\mathbf{1})$ towards 2 -amino- $4 H$-pyrans 7.

\subsection{Excess of ethyl cyanoacetate 5}

The formation of triethylammonium salts $\mathbf{6}$ and ethyl 2-amino$4 H$-pyran-3-carboxylates 7 was considered by us as two competitive processes (Scheme 2). Previously, the application of ethyl cyanoacetate excess allowed us to shift the equilibrium, established in the reaction mixture, towards the formation of the target ethyl 2-amino- $4 H$-pyran-3-carboxylates. ${ }^{9,10 b}$ According to this, it was logical to use an excess of 5 in the studied reaction. Thus, the utilization of 2 equiv. of $5\left(1\right.$ equiv. of $\mathrm{Et}_{3} \mathrm{~N}$, reflux for $4 \mathrm{~h}$ ) also led to the isolation of salt $6 \mathbf{6}$ in $24 \%$ yield. Applying of 3 equiv. of 5 under the previous reaction conditions allowed us to isolate the mixture of the initial 1,2-benzoxathiin-4(3H)one 2,2-dioxide (1), arylidene 8a and target 2-amino- $4 H$-pyran-3carboxylate $7 \mathbf{a}$ in an approximate molar ratio of $1: 1: 1$ according to ${ }^{1} \mathrm{H}$ NMR spectroscopy. It might have seemed like the reaction did not complete during the heating time. In this regard, the above-mentioned interaction was carried out for $10 \mathrm{~h}$ in similar conditions, but the mixture of equimolar amounts of 2-amino- $4 \mathrm{H}$-pyran-3-carboxylate $7 \mathbf{a}$ and triethylammonium salt $\mathbf{6 a}$ was isolated. The same mixture was also isolated when a 4 -fold excess of $\mathbf{5}$ was used. This may serve as evidence of an equilibrium established between the products $6 \mathbf{6}$ and $7 \mathbf{a}$ in the course of the reaction performed. When a 5 -fold or more excess of $\mathbf{5}$ was introduced to the reaction, no solid was isolated from the mixture.

\subsection{Variation of catalysts}

Literature data reveal that a wide range of basic catalysts can be applied in similar interactions that result in high yields of 2amino- $4 H$-pyrans irrespective of the nature of the 1,3-dicarbonyl and nitrile components. ${ }^{21}$ However, it was shown that, in certain instances, the catalyst is a crucial factor directing the reaction towards unexpected outcomes. ${ }^{16}$ We selected 4-dimethylaminopyridine (DMAP), DBU and ammonium acetate as the tested catalysts. The first was chosen since in the case of the $\mathrm{N}$ containing analog of 1, DMAP appeared to be an effective catalyst in the synthesis of some 3-ethoxycarbonyl derivatives of 2-amino- $4 \mathrm{H}$-pyrans ${ }^{9}$ and DBU was selected because of its high basicity. However, reflux for $4 \mathrm{~h}$ of the alcohol solution of benzoxathiinone 1 with benzaldehyde (2a) and 3 equiv. of 5 (Scheme 2) under the catalysis of DMAP (1 equiv.) led to the isolation of 4-dimethylaminopyridinium salt $6 \mathbf{6 P}$ in $37 \%$ yield. A similar result was also obtained when DBU was used as a catalyst in the reaction and salt $6 \mathbf{a D}$ was isolated with $43 \%$ yield. In the case of ammonium acetate utilization ( 1 equiv., $\mathrm{AcOH}$, reflux for $4 \mathrm{~h}$ ), analysis of the reaction mixture by a ${ }^{1} \mathrm{H}$ NMR method showed the presence of the starting benzoxathiinone 1 together with ethyl 2-amino- $4 \mathrm{H}$-pyran-3-carboxylate $7 \mathbf{a}$ and arylidene $\mathbf{8 a}$.

\subsection{Two-component approach}

According to the above-mentioned, variation of the reaction conditions did not provide us with the desired outcome. Because of this, we focused our attention on the two-component format of ethyl 2-amino- $4 \mathrm{H}$-pyran-3-carboxylates 7 synthesis based on the reaction of the Knoevenagel products 8 with benzoxathiinone 1 (Scheme 2). One could assume that such an approach would allow us to avoid the formation of salts $\mathbf{6}$ (as the result of a possible direct interaction of $\mathbf{1}+2$ ) and additionally would help to clarify some aspects of the reaction mechanism. However, even such a stepwise format under the catalysis of $\mathrm{Et}_{3} \mathrm{~N}$ (1 equiv., reflux for $4 \mathrm{~h}$ ) led to the isolation of 


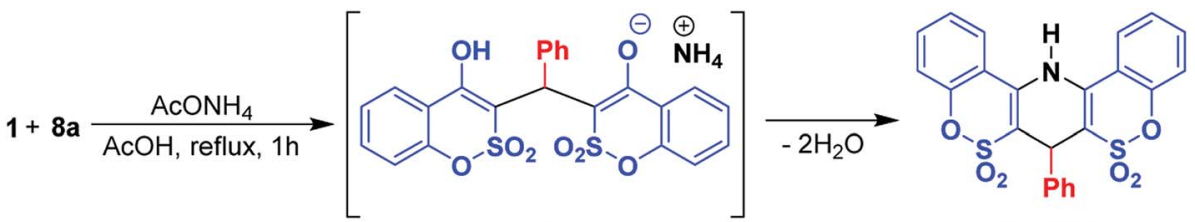

Scheme 3 Formation of 7-phenyl-7,14-dihydrobenzo[5,6][1,2] oxathiino[4,3-b]benzo[5,6][1,2] oxathiino[3,4-e]pyridine 6,6,8,8-tetraoxide 9a.

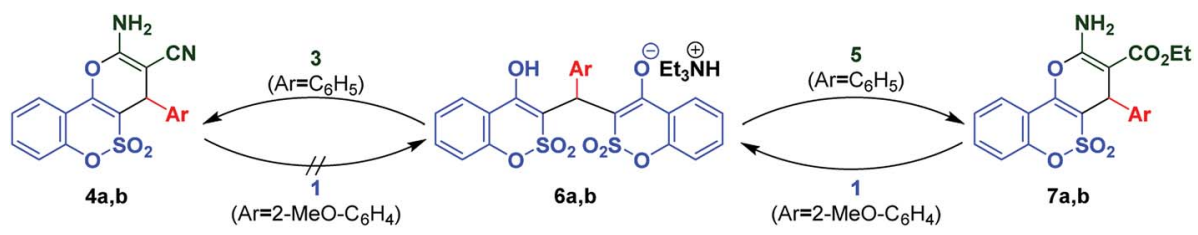

Scheme 4 The study of the mutual transformation ( $\mathrm{EtOH}, \mathrm{Et}_{3} \mathrm{~N}$, reflux for $\left.4 \mathrm{~h}\right)$ of the compounds 4, 6 and 7.

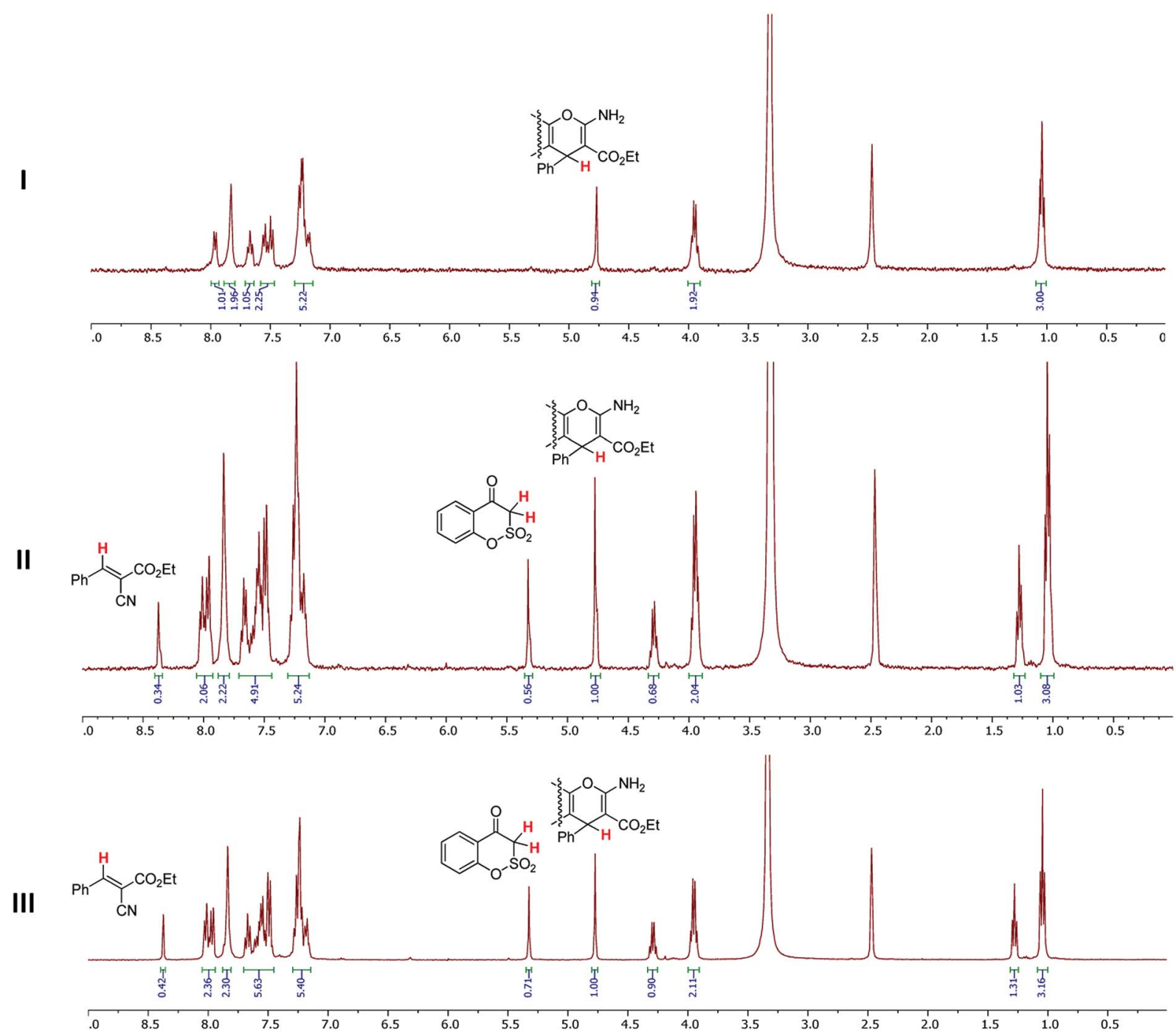

Fig. $3{ }^{1} \mathrm{H}$ NMR spectra of 7a (DMSO-d , r.t., analyzed by MestReNova v12.0.2) measured: I - immediately after dissolving; II - in $24 \mathrm{~h}$ after dissolving; III - in $48 \mathrm{~h}$ after dissolving. 


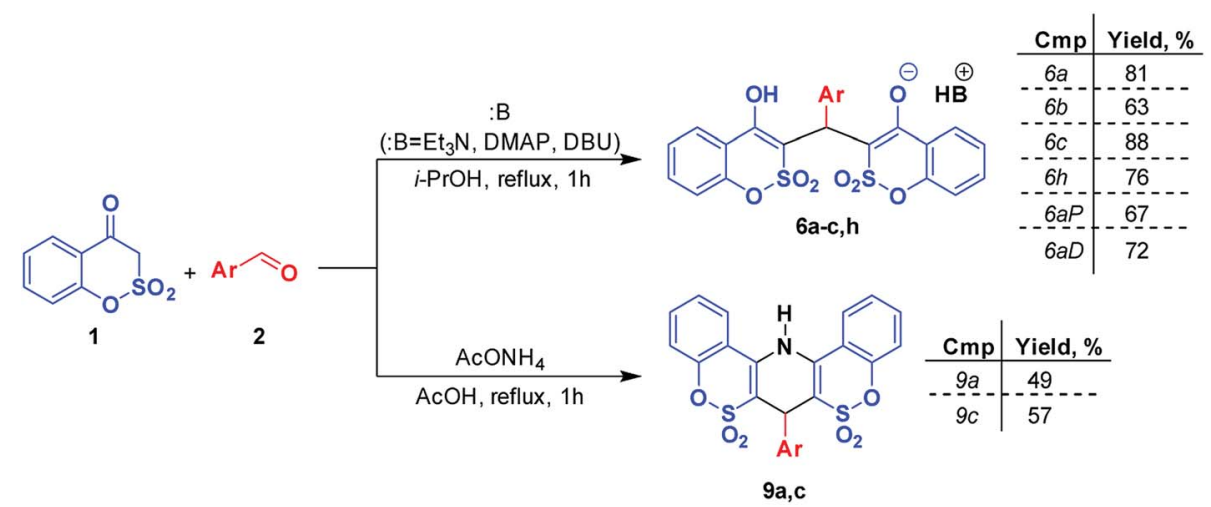

Scheme 5 Preparative methods for the synthesis of ammonium salts 6 and 1,4-dihydropyridines 9 .

triethylammonium salt 6 a in $78 \%$ yield. The application of DBU instead of $\mathrm{Et}_{3} \mathrm{~N}$ in the two-component format led to the corresponding salt $6 \mathbf{a}$ (yield $-33 \%$ ) even at room temperature. These facts suggest that the reaction proceeds through the formation of the highly reactive arylidene $\mathbf{G}$ formed by Michael cleavage of the initially generated adduct $\mathbf{H}$ (Scheme 2). Intermediate $\mathbf{G}$ subsequently adds benzoxathiinone $\mathbf{1}$ in the presence of a base giving salt 6. Apparently, the transformation of salt 6 into ethyl 2-amino- $4 H$-pyran-3-carboxylate 7 is also possible by means of the reverse reactions.

The use of ammonium acetate provided us with another new derivative of 1,2-benzoxathiine 2,2-dioxide and a novel product for such types of interactions - 7-phenyl-7,14-dihydrobenzo[5,6][1,2] oxathiino[4,3- $b]$ benzo[5,6][1,2] oxathiino [3,4-e]pyridine 6,6,8,8-tetraoxide (9a) when a mixture of 1 and 8a was refluxed in $\mathrm{AcOH}$ for $1 \mathrm{~h}$ in the presence of 1 equiv. of ammonium acetate (Scheme 3). Apparently, the initially formed ammonium salt under the reaction conditions loses 2 molecules of water and cyclizes into 1,4-dihydropyridine 9a.

The interactions, presented in Scheme 2, resulted into salts 6 and/or 2-amino-4H-pyrans 7. Thus, there is a clear distinction between the reactivity of malononitrile and ethyl cyanoacetate in the three-component reactions. It is possible that these differences are associated with the different $\mathrm{p} K_{\mathrm{a}}$ values of the $\mathrm{CH}_{2}$ moiety of the two compounds; one other possible reason for this may be differences in the reactivity of the double bond in the resultant Knoevenagel condensation products. Taking into account the obtained results, we may conclude that the formation of ammonium salts 6 dominates over the formation of ethyl 2-amino- $4 H$-pyran-3-carboxylates 7 and even the application of ethyl cyanoacetate (5) excess, as well as that the use of a twocomponent format of the synthesis did not allow us to manage the selectivity of the reaction. One could assume that the generation of stable ammonium salts continuously pushes the reaction equilibrium towards their formation. Because of this we decided to check the possibility that salts 6 could be converted into pyrans 7 (Scheme 4). This research we carried out together with the study of the transformation $6 \rightarrow 4$, since the utilization of malononitrile (3) unambiguously led to 2-amino-4H-pyran-3carbonitriles 4 . The reverse transformations $7 \rightarrow 6$ and $4 \rightarrow 6$ were also investigated. It was found that the interaction of salt $\mathbf{6 a}$ with malononitrile (3) or ethyl cyanoacetate (5) resulted in the isolation of a mixture of the starting salt and appropriate pyran 4a or 7a. At the same time, only the 3-ethoxycarbonyl derivative $7 \mathbf{b}$ was able to be converted into salt $\mathbf{6 b}$ when reacted with $\mathbf{1}$; in the case of the 3 -cyano derivative $\mathbf{4 b}$ reaction with $\mathbf{1}$, only the starting materials were recovered. These results explain the facts that the 2-amino- $4 H$-pyran-3-carbonitriles 4 are formed as single products in the three-component interaction when malononitrile (3) was used (Table 1), whereas the application of ethyl

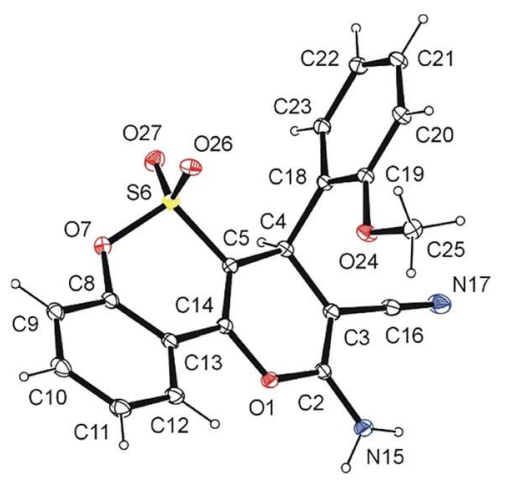

$4 b$

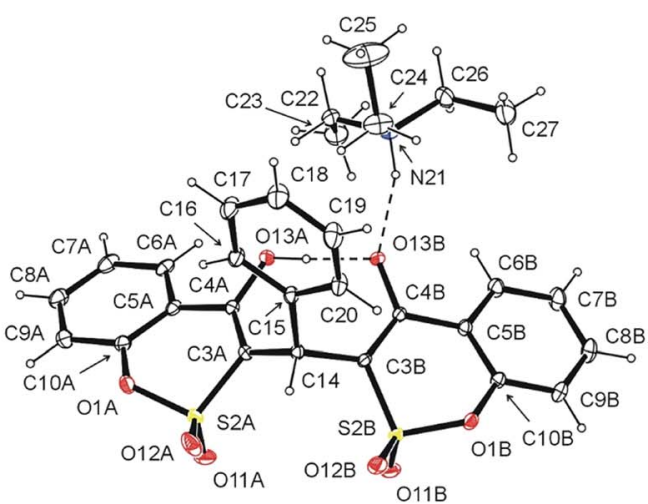

$6 a$

Fig. 4 Molecular structure (ORTEP plot) of 4b (deposition no. CCDC 1585657) and 6a (deposition no. CCDC 1585656) showing the atomic labeling scheme. Non- $\mathrm{H}$ atoms are drawn as $30 \%$ probability displacement ellipsoids and $\mathrm{H}$ atoms are drawn as spheres of an arbitrary size. 
cyanoacetate (5) resulted in the isolation of the product mixture (Scheme 2).

In respect of the interconversions established, the formation of relatively unstable salts in the course of the reaction might shift the equilibrium towards 2-amino- $4 H$-pyrans 7 . To check this hypothesis, we introduced into the two-component reaction such a base as sodium acetate. Its application in the reaction of 1 with 8a allowed us to isolate pure target ethyl 2-amino-4-phenyl- $4 \mathrm{H}$ pyran-3-carboxylate $7 \mathbf{a}$ in $24 \%$ yield. A very unexpected result was occasionally gathered by us during the measurement of the ${ }^{1} \mathrm{H}$ NMR spectra of $7 \mathbf{a}$. This essential fact referred to the stability of ethyl 2-amino- $4 H$-pyran-3-carboxylates 7 (Fig. 3). ${ }^{1} \mathrm{H}$ NMR spectra of $7 \mathrm{a}$ recorded at $24 \mathrm{~h}$ and $48 \mathrm{~h}$ showed the presence of a high percentage of the initial benzoxathiinone 1 and ethyl $\alpha$-cyano- $\beta$ phenylacrylate (8a) increasing over time. Apparently, DMSO induces the retro-Michael cleavage of 7a, most probably as a result of its weak basic properties. ${ }^{22}$

Additionally, preparative two-component approaches were worked out for the synthesis of salts 6 and 1,4-dihydropyridines 9. These routes are based on the interaction of benzoxathiinone 1 with benzaldehydes 2 in different reaction conditions (Scheme 5).

Structural features of the synthesized 2-amino-4-aryl- $4 \mathrm{H}$ pyrano[3,2-c][1,2]benzoxathiine-3-carbonitrile 5,5-dioxides and triethylammonium 3-((4-hydroxy-2,2-dioxidobenzo[e][1,2] oxathiin-3-yl)(aryl)methyl)benzo[e][1,2]oxathiin-4-olate 2,2dioxides were confirmed by X-ray crystallographic analysis of example compounds $\mathbf{4 b}$ and $6 \mathbf{a}$ (Fig. 4 ).

\section{Conclusion}

The three-component interaction of 1,2-benzoxathiin-4(3H)-one 2,2-dioxide (1) with benzaldehydes and active methylene nitriles is a complicated process. Its outcome was controlled by the nitrile nature. In the case of malononitrile (3), the products formed were solely 2-amino- $4 H$-pyran-3-carbonitriles 4 . When ethyl cyanoacetate (5) was used under basic catalysis, the reaction led to the product mixture as a result of a number of equilibrium processes existing between the starting compounds, ethyl 3-aryl-2-cyanoacrylates 8, ethyl 2-amino-4aryl- $4 H$-pyran-3-carboxylates 7 and triethylammonium salts 6 . In this instance, the composition of the isolated mixture strongly depended on the reaction conditions applied and relative solubility of the components. The low stability established for ethyl 2-amino-4-aryl-4H-pyran-3-carboxylates 7 and the difficulties of obtaining them motivated us to search for a new effective route towards these condensed derivatives, for reasons including their pharmacological attractiveness. The study of the three-component interaction involving ethyl cyanoacetate (5) allowed us to isolate for the first time the ammonium salts 6 and 1,4-dihydropyridines 9.

\section{Experimental}

4.1. Synthesis of 1,2-benzoxathiin-4(3H)-one 2,2-dioxide (1)

To a cooled to $0{ }^{\circ} \mathrm{C}$ vigorously stirred mixture of methyl salicylate $(64.50 \mathrm{~mL}, 0.5 \mathrm{~mol})$, triethylamine $(76.51 \mathrm{~mL}, 0.55 \mathrm{~mol})$ and diethyl ether $(700 \mathrm{~mL})$, methanesulfonyl chloride $(42.35 \mathrm{~mL}$, $0.55 \mathrm{~mol}$ ) was added dropwise. The temperature was slowly raised to $15-20{ }^{\circ} \mathrm{C}$ and the resulting mixture was stirred for $24 \mathrm{~h}$. After the completion of this time, water $(500 \mathrm{~mL})$ was added to the suspension and the organic layer was separated. The ethereal solution was successively washed with a $1 \mathrm{M}$ solution of sodium bicarbonate $(100 \mathrm{~mL})$ and water $(200 \mathrm{~mL})$, dried over $\mathrm{Na}_{2} \mathrm{SO}_{4}$ and concentrated in vacuo. The residue obtained was methyl 2-((methylsulfonyl)oxy)benzoate as a light-yellow solid (mp 35-37 ${ }^{\circ} \mathrm{C}$ ) which was used in the next step without additional purification.

A solution of methyl 2-((methylsulfonyl)oxy)benzoate (46.0 g, $0.2 \mathrm{~mol})$ in dry DMF $(100 \mathrm{~mL})$ was added dropwise to a suspension of $\mathrm{NaH}(8.8 \mathrm{~g}$ of $60 \%$ suspension in mineral oil, $0.22 \mathrm{~mol})$ in dry DMF $(70 \mathrm{~mL})$ at $0{ }^{\circ} \mathrm{C}$. After completion of the hydrogen evolution, the mixture was additionally stirred for $1.5 \mathrm{~h}$ at $0{ }^{\circ} \mathrm{C}$ and to the resulted suspension diluted $\mathrm{HCl}$ was added to achieve $\mathrm{pH} 1$. The precipitate of 1,2-benzoxathiin4(3H)-one 2,2-dioxide (1) was collected by filtration, dried in air and recrystallized from ethanol. The total yield of 1 was $64 \%$.

White crystalline powder; mp 87-89 ${ }^{\circ} \mathrm{C}$ (from EtOH) (lit. 2a 89-91 ${ }^{\circ} \mathrm{C}$ ); anal. calcd for $\mathrm{C}_{18} \mathrm{H}_{12} \mathrm{~N}_{2} \mathrm{O}_{4} \mathrm{~S}: \mathrm{C}, 48.48 ; \mathrm{H}, 3.05 ; \mathrm{S}$, 16.18. Found, \%: C 48.61; H 3.11; S, $16.04 ;{ }^{1} \mathrm{H}$ NMR $(400 \mathrm{MHz}$, DMSO-d $)^{23}: \delta(\mathrm{ppm}) 8.00(\mathrm{~d}, J=7.19 \mathrm{~Hz}, 1 \mathrm{H}, \mathrm{Ar}), 7.85(\mathrm{t}, J=$ $7.05 \mathrm{~Hz}, 1 \mathrm{H}, \mathrm{Ar}$ ), $7.42-7.56$ (m, 2H, Ar), 5.34 (br. s., $2 \mathrm{H}, \mathrm{CH}_{2}$ ).

\subsection{General procedure for the synthesis of 2-amino-4-aryl-4H-} pyrano[3,2-c] [1,2]benzoxathiine-3-carbonitrile 5,5-dioxides (4a-i)

To a solution of 1,2-benzoxathiin-4(3H)-one 2,2-dioxide (1) $(0.198 \mathrm{~g}, 0.001 \mathrm{~mol})$, the appropriate aromatic aldehyde $\mathbf{2 a}-\mathbf{i}$ (0.001 mol) and malononitrile (3) (0.066 g, $0.001 \mathrm{~mol})$ in ethanol $(10 \mathrm{~mL})$, triethylamine $(0.014 \mathrm{~mL}, 0.0001 \mathrm{~mol})$ was added. The mixture was refluxed for $1 \mathrm{~h}$ and cooled to room temperature. The resulting precipitates of $\mathbf{4 a - i}$ were filtered off, washed with ethanol, dried in air and recrystallized from the mixture EtOHDMF $(5: 1, \mathrm{v} / \mathrm{v})$.

\subsection{Preparative procedure for the synthesis of} triethylammonium 3-((4-hydroxy-2,2-dioxidobenzo[e][1,2] oxathiin-3-yl)(aryl)methyl)benzo[ $e][1,2]$ oxathiin-4-olate 2,2dioxides $(6 a-c, h)^{24}$

To a solution of 1,2-benzoxathiin-4(3H)-one 2,2-dioxide (1) $(0.198 \mathrm{~g}, 0.001 \mathrm{~mol})$ and the appropriate aromatic aldehyde 2ac,h $(0.0005 \mathrm{~mol})$ in propan-2-ol $(10 \mathrm{~mL})$, triethylamine $(0.07 \mathrm{~mL}$, $0.0005 \mathrm{~mol}$ ) was added. The solution was stirred for $1 \mathrm{~h}$ under reflux. The obtained precipitates of $\mathbf{6 a}-\mathbf{c}, \mathbf{h}$ were filtered off, washed with propan-2-ol, dried in air and recrystallized from ethanol.

\subsection{Synthesis of ethyl 2-amino-4-phenyl-4H-pyrano[3,2-c] $[1,2]$ benzoxathiine-3-carboxylate 5,5-dioxides (7)}

4.4.1 Ethyl 2-amino-4-phenyl-4H-pyrano[3,2-c][1,2]benzoxathiine3-carboxylate 5,5-dioxide (7a). Solution of 1,2-benzoxathiin-4(3H)one 2,2-dioxide (1) (0.198 g, $0.001 \mathrm{~mol}$ ), ethyl 2-cyano-3phenylacrylate $(\mathbf{8 a})(0.201 \mathrm{~g}, 0.001 \mathrm{~mol})$ and sodium acetate $(0.2 \mathrm{~g})$ in ethanol $(10 \mathrm{~mL})$ was refluxed for $24 \mathrm{~h}$ and cooled to 
room temperature. The formed precipitate was filtered off, washed successively with water and ethanol and dried in air.

4.4.2 Ethyl 2-amino-4-(2'-methoxyphenyl)-4H-pyrano[3,2-c] $[1,2]$ benzoxathiine-3-carboxylate 5,5 -dioxide (7b). A solution of 1,2-benzoxathiin-4(3H)-one 2,2-dioxide (1) (0.198 g, $0.001 \mathrm{~mol})$, 2-methoxybenzaldehyde (2b) (0.136 g, $0.001 \mathrm{~mol})$, ethyl cyanoacetate (5) (0.113 g, $0.001 \mathrm{~mol})$ and triethylamine $(0.14 \mathrm{~mL}$, $0.001 \mathrm{~mol})$ in ethanol $(10 \mathrm{~mL})$ was refluxed for $4 \mathrm{~h}$ and cooled to room temperature. The formed precipitate of $7 \mathbf{b}$ was filtered off, washed with cooled to $0{ }^{\circ} \mathrm{C}$ ethanol and dried in air.

\subsection{Preparative procedure for the synthesis of 7-aryl-7,14- dihydrobenzo $[5,6][1,2]$ oxathiino $[4,3-b]$ benzo $[5,6][1,2]$ oxathiino[3,4-e]pyridine 6,6,8,8-tetraoxides (9)}

A solution of 1,2-benzoxathiin-4(3H)-one 2,2-dioxide (1) $(0.396 \mathrm{~g}, 0.002 \mathrm{~mol})$, the appropriate aromatic aldehyde 2a,c $(0.001 \mathrm{~mol})$ and ammonium acetate $(0.77 \mathrm{~g}, 0.01 \mathrm{~mol})$ in $\mathrm{AcOH}$ $(10 \mathrm{~mL})$ was refluxed for $1 \mathrm{~h}$ and cooled to room temperature. The resulting precipitates of 9a,c were filtered off, washed successively with $\mathrm{AcOH}$ and water and dried in air.

\section{Conflicts of interest}

There are no conflicts to declare.

\section{Acknowledgements}

The authors are thankful to Mr Eugene S. Gladkov (SSI "ISC" NASU) for his support in obtaining the spectroscopic data for the compounds presented in this paper.

\section{Notes and references}

1 For some examples, see: (a) W. Löwe and C. Müller-Menke, Arch. Pharm., 1985, 318, 954-956; (b) W. Löwe and C. Müller-Menke, Arch. Pharm., 1988, 321, 755-756; (c) W. Löwe, P. Jeske and A. Kradepohl, J. Heterocycl. Chem., 1988, 25, 699-701.

2 (a) V. R. Arava, U. B. R. Siripalli, V. Nadkarni and R. Chinnapillai, Beilstein J. Org. Chem., 2007, 3, 20; (b) C. Peixoto, P. Laurin, M. Klich, C. Dupuis-Hamelin, P. Mauvais, P. Lassaigne, A. Bonnefoy and B. Musicki, Tetrahedron Lett., 2000, 41, 1741-1745.

3 C. Hulme and V. Gore, Curr. Med. Chem., 2003, 10, 51-80.

4 (a) Y. V. Sedash, N. Y. Gorobets, V. A. Chebanov, I. S. Konovalova, O. V. Shishkin and S. M. Desenko, RSC $A d v ., \quad 2012, \quad 2$, 6719-6728; (b) E. A. Muravyova, S. M. Desenko, R. V. Rudenko, S. V. Shishkina, O. V. Shishkin, Y. V. Sen'ko, E. V. Vashchenko and V. A. Chebanov, Tetrahedron, 2011, 67, 9389-9400.

5 V. A. Chebanov and S. M. Desenko, Chem. Heterocycl. Compd., 2012, 48, 566-583.

6 C. W. Thornber, Chem. Soc. Rev., 1979, 8, 563-580.

7 N. Au and A. E. Rettie, Drug Metab. Rev., 2008, 40, 355-375.
8 (a) I. V. Ukrainets, L. A. Petrushova, S. P. Dzyubenko and G. Sim, Chem. Heterocycl. Compd., 2014, 50, 103-110; (b) D. A. Lega, N. I. Filimonova, I. A. Zupanets, S. K. Shebeko, V. P. Chernykh and L. A. Shemchuk, J. Org. Pharm. Chem., 2016, 14, 3-11.

9 D. A. Lega, N. Y. Gorobets, V. P. Chernykh, S. V. Shishkina and L. A. Shemchuk, RSC Adv., 2016, 6, 16087-16099.

10 (a) D. A. Lega, V. P. Chernykh, L. Zaprutko, A. K. Gzella and L. A. Shemchuk, Chem. Heterocycl. Compd., 2017, 53, 219229; (b) D. A. Lega, V. P. Chernykh and L. A. Shemchuk, J. Org. Pharm. Chem., 2016, 14, 6-16.

11 A.-G. E. Amr, A. M. Mohamed, S. F. Mohamed, N. A. AbdelHafez and A. E.-F. G. Hammam, Bioorg. Med. Chem., 2006, 14, 5481-5488.

12 P. Paliwal, S. Jetti and S. Jain, Med. Chem. Res., 2013, 22, 2984-2990.

13 D. Kumar, V. B. Reddy, S. Sharad, U. Dube and S. Kapur, Eur. J. Med. Chem., 2009, 44, 3805-3809.

14 C. W. Smith, J. M. Bailey, M. E. J. Billingham, S. Chandrasekhar, C. P. Dell, A. K. Harvey, C. A. Hicks, A. E. Kingston and G. N. Wishart, Bioorg. Med. Chem. Lett., 1995, 5, 2783-2788.

15 C. F. Schwender and B. R. Sunday, US pat. US4116961A, 1978.

16 Y. M. Litvinov and A. M. Shestopalov, in Adv. Heterocycl. Chem., ed. A. R. Katritzky, Academic Press, 2011, vol. 103, pp. 175-260.

17 (a) K. Azizi and A. Heydari, RSC Adv., 2014, 4, 6508-6512; (b) M.-J. Yao, Z. Guan and Y.-H. He, Synth. Commun., 2013, 43, 2073-2078; (c) J. Safaei-Ghomi, R. Teymuri, H. ShahbaziAlavi and A. Ziarati, Chin. Chem. Lett., 2013, 24, 921-925; (d) G. H. Mahdavinia, M. Mirzazadeh and B. Notash, Tetrahedron Lett., 2013, 54, 3487-3492.

18 L. Jalili-Baleh, N. Mohammadi, M. Khoobi, L. Ma'mani, A. Foroumadi and A. Shafiee, Helv. Chim. Acta, 2013, 96, 1601-1609.

19 B. Maheshwar Rao, G. N. Reddy, T. V. Reddy, B. L. A. P. Devi, R. B. N. Prasad, J. S. Yadav and B. V. S. Reddy, Tetrahedron Lett., 2013, 54, 2466-2471.

20 A. Thakur, M. Tripathi, U. C. Rajesh and D. S. Rawat, $R S C$ Adv., 2013, 3, 18142-18148.

21 G. Brahmachari, in Green Synthetic Approaches for Biologically Relevant Heterocycles, ed. G. Brahmachari, Elsevier, Boston, 2015, pp. 185-208.

22 W. S. MacGregor, Ann. N. Y. Acad. Sci., 1967, 141, 3-12.

23 According to ${ }^{1} \mathrm{H}$ NMR spectrum, 1,2-benzoxathiin-4(3H)-one 2,2-dioxide (1) in DMSO- $\mathrm{d}_{6}$ solution exists in two tautomeric forms - 4-oxo form and 4-hydroxy (enol) form in aproximate molar ratio $10: 1$.

24 The same synthetic procedure was applied for the preparation of 4-(dimethylamino)pyridin-1-ium 6aP and 3,4,5,6,7,8,9,10-octahydro-2 $H$-pyrimido[1,2-a]azepin-5-ium 6aD salts using DMAP and DBU, respectively. 